SCIENTIFIC LETTER

\title{
Prolonged exercise should be considered alongside typical symptoms of acute myocardial infarction when evaluating increases in cardiac troponin $\mathrm{T}$
}

\author{
R E Shave, G P Whyte, K George, D C Gaze, P O Collinson
}

Heart 2005;91:1219-1220. doi: 10.1136/hrt.2004.046052

$\mathrm{F}$ ollowing the recommendations of the European Society of Cardiology and the American College of Cardiology in 2000, cardiac troponins $\mathrm{T}$ ( $\mathrm{cTnT}$ ) and I are now integral to the diagnosis of non-ST elevation myocardial infarction. Although discussion is ongoing regarding the appropriate cut off for cTnT, the use of cTnT in the diagnosis of acute myocardial infarction (AMI) has greatly enhanced diagnostic sensitivity and further improved risk stratification. Cardiac troponin determinations are not required for diagnosis or treatment of classic ST segment elevation myocardial infarction, as thrombolysis is administered solely on the basis of ECG criteria. It is noteworthy, however, that within a clinical population any rise in CTnT above the detection limit of the assay is related to an increased risk of death. ${ }^{2}$ Recent studies of highly trained endurance athletes completing ultraendurance exercise have reported isolated increases of cardiac troponins in the absence of acute coronary symptoms. ${ }^{3}$ To date, only limited investigation has examined cTnT after more readily accessible and increasingly popular events such as the London Marathon in less well trained people. Accordingly, we investigated the impact of running the London Marathon on circulating cTnT in a heterogeneous cohort of non-elite runners.

\section{METHODS}

Data were collected at the 2002 and 2003 London Marathons. Following local ethics approval, we obtained written informed consent from 72 runners (mean (SD) age 35 (9) years) to participate in the study. On the basis of a self reported history all participants were apparently healthy, free from cardiovascular disease, and non-medicated. Whole blood samples were drawn, a standard 12 lead ECG was recorded, and standard echocardiography was performed 24 hours before the race and within 30 minutes of race completion. Indices of left ventricular diastolic (ratio of early to late ventricular filling (E:A)) and systolic (end systolic volume-pressure relation) function were calculated. Serum samples were analysed for cTnT with the third generation immunoassay (Roche Diagnostics, Lewes, UK). The assay imprecision was $5.5 \%$ at $0.32 \mu \mathrm{g} / \mathrm{l}$ and $5.4 \%$ at $6 \mu \mathrm{g} / \mathrm{l}$, and had a detection limit of $0.01 \mu \mathrm{g} / \mathrm{l}$ and an upper limit of $25 \mu \mathrm{g} / \mathrm{l}$. Data were analysed for normality by the KolmogorovSmirnov test. Age, completion time, and functional indices were all normally distributed ( $\mathrm{p}>0.05)$. The cTnT data were, however, skewed $(\mathrm{p}<0.05)$, probably because of the large number of samples below the assay detection limit. Owing to the abnormal distribution of the cTnT data, analyses was completed by determining the percentage of subjects with a rise in cTnT above the current suggested AMI cut off $(0.05 \mu \mathrm{g} / \mathrm{l})^{1}$ and a number of previously suggested AMI cut offs $(0.01,0.03$, and $0.1 \mu \mathrm{g} / \mathrm{l}) .{ }^{1}$ Pearson correlation coefficients were calculated between increases in cTnT and age, completion time, and indices of left ventricular function.

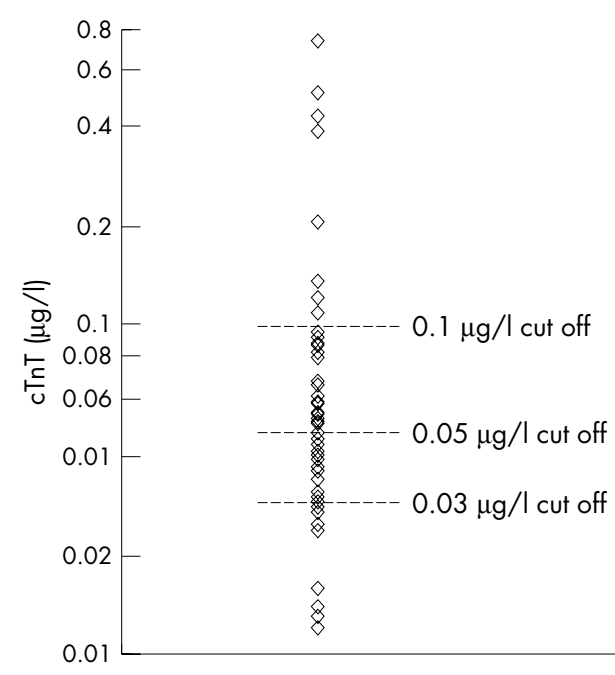

Figure 1 Positive cardiac troponin T (cTnT) samples after the London marathon (scale is log plotted because of the data spread).

\section{RESULTS}

Mean (SD) completion time of the marathon was 253 (48) minutes. Before the marathon all $\mathrm{CTnT}$ samples were below the $0.01 \mu \mathrm{g} / \mathrm{l}$ detection limit of the assay. After race completion 56 subjects $(78 \%)$ had cTnT concentrations above $0.01 \mu \mathrm{g} / \mathrm{l}$ (range $0.012-0.733 \mu \mathrm{g} / \mathrm{l}$ ). Of these, 42 (58\%) had concentrations above $0.03 \mu \mathrm{g} / \mathrm{l}, 26(36 \%)$ above the current AMI cut off $(0.05 \mu \mathrm{g} / \mathrm{l})$, and eight $(11 \%)$ above $0.1 \mu \mathrm{g} / \mathrm{l}$. Figure 1 presents a scatter plot of the positive cTnT samples. No ECG evidence of ischaemia was observed before or after race completion. After completion of the marathon systolic function did not change significantly; however, a significant reduction in E:A ( $1.7(0.4) v 1.2(0.3))$ showing altered left ventricular filling was observed $(\mathrm{p}<0.05)$. Increases in $\mathrm{cTnT}$ after the marathon were not related to age $(r=0.15)$, completion time $(r=0.01)$, or changes in E:A $(r=0.16)$ $(\mathrm{p}>0.05)$.

\section{DISCUSSION}

Within the present study minimally increased serum concentrations above $0.01 \mu \mathrm{g} / \mathrm{l}$ of cTnT were widely observed after marathon running in non-elite runners in the absence of any ECG abnormalities. In addition cTnT concentrations exceeded all or some of the previously examined cTnT cut off criteria. $^{1}$ Left ventricular filling was significantly different

Abbreviations: AMl, acute myocardial infarction; $E: A$, ratio of early to late ventricular filling; cTnT, cardiac troponin T 
after race completion; however, no relation was observed between CTnT rise and altered diastolic filling. The significance of an increased cTnT after prolonged exercise may be confused by clinical findings, such as dyspnoea, chest tightness, and possible ECG abnormalities ${ }^{4}$ occasionally $^{2}$ observed in athletes after prolonged exercise. Accordingly, caution is advised on the use of cTnT cut off criteria in the diagnosis of AMI in patients who have recently completed a bout of prolonged exercise. Although not assessed within the present study, previous studies of highly trained athletes suggest that, 24 hours after completion of a prolonged bout of exercise, cardiac troponin concentrations return below the lower limit of detection. ${ }^{3}$ Further, previous research indicates that changes in cardiac function following prolonged exercise are also transient. ${ }^{5}$

The aetiology of exercise induced cTnT release and alterations in cardiac function is unclear. However, the widespread increases in CTnT within the present study suggest that the phenomenon is probably physiological. Increased coronary artery shear stress, oxidative stress, and ischaemia have been suggested as potential mechanisms, all of which are mediated by prolonged exercise. ${ }^{5}$ The relatively low concentrations of cTnT observed in the present study may result from leakage from the cytosol as opposed to the breakdown of the contractile apparatus. Cytosolic release of cTnT might result from membrane damage propagated by any of the conditions outlined previously. Work is warranted to elucidate further the mechanisms responsible for both exercise induced cTnT release and exercise induced changes in diastolic function. Moreover, the possible long term consequences of exercise induced cTnT release need to be examined. In a clinical setting, elevations in CTnT should be assessed with reference to prolonged exercise as well as typical symptoms of AMI.

\section{Authors' affiliations}

R E Shave, Brunel University, Uxbridge, Middlesex, UK G P Whyte, Olympic Medical Institute, Northwick Park Hospital, Harrow, Middlesex, UK

K George, Liverpool John Moores University, Liverpool, UK D C Gaze, P O Collinson, St Georges Healthcare NHS Trust, Tooting, London, UK

Competing interest statement: All authors declare that there is no competing interest and therefore have nothing to declare.

We acknowledge the support of Cardiac Risk in the Young.

Correspondence to: Dr Robert E Shave, Brunel University, Uxbridge, Middlesex UB8 3PH, UK; rob.shave@brunel.ac.uk

Accepted 29 October 2004

\section{REFERENCES}

1 Collinson PO, Stubbs PJ, Kessler A-C. Multicentre evaluation of the diagnostic value of cardiac troponin T, CK-MB mass, and myoglobin for assessing patients with suspected acute coronary syndromes in routine clinical practice. Heart 2003;89:280-6.

2 James S, Armstrong P, Califf R, et al. Troponin T levels and risk of 30-day outcomes in patients with the acute coronary syndrome: prospective verification in the GUSTO-IV trial. Am J Med 2003; 115:178-84.

3 Neumayr G, Pfister R, Mitterbauer G, et al. Effect of the "race across the alps" in elite cyclists on plasma cardiac troponins I and T. Am J Cardiol 2002:89:484-6.

4 Pelliccia A, Maron BJ, Culasso F, et al. Clinical significance of abnormal electrocardiographic patterns in trained athletes. Circulation 2000;102:278-84.

5 Dawson E, George K, Shave R, et al. Does the human heart fatigue subsequent to prolonged exercise? Sports Med 2003;33:365-80.

\section{FROM BMJ JOURNALS}

\section{Overweight and obesity and weight change in middle aged men: impact on cardiovascular disease and diabetes}

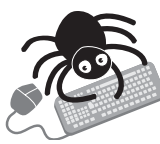

Please visit the Heart website [www.heartin. com] for a link to the full text of this article.
S Goya Wannamethee, A Gerald Shaper, Mary Walker

Context: The benefit of weight reduction for cardiovascular disease (CVD) outcomes remains uncertain.

Objective: To examine the effects of baseline body mass index on major CVD outcomes and diabetes over a 20 year follow up, and of weight change in the first five years over the subsequent 15 years.

Design and setting: A prospective study of British men followed up for 20 years.

Participants: Men aged 40-59 years with no diagnosis of CVD or diabetes $(n=7176)$ of whom 6798 provided full information on weight change five years later.

Outcome measures: Major CVD events (fatal and non-fatal myocardial infarction and stroke, angina, "other" CVD deaths) and diabetes.

Results: During the 20 year follow up there were 1989 major CVD events and 449 incident cases of diabetes in the 7176 men. Risk of major CVD and diabetes increased significantly with increasing overweight and obesity. During the 15 year follow up, weight gain was associated with increased risk of CVD and diabetes. Weight loss was associated with lower risk of diabetes than the stable group irrespective of initial weight. No significant cardiovascular benefit was seen for weight loss in any men, except possibly in considerably overweight (BMI $27.5-29.9 \mathrm{~kg} / \mathrm{m}^{2}$ ) younger middle aged men $(\mathrm{RR}=0.42 ; 95 \% \mathrm{CI} 0.22$ to $0.81)$.

Conclusion: Long term risk of CVD and diabetes increased significantly with increasing overweight and obesity. Weight loss was associated with significant reduction in risk of diabetes but not CVD, except possibly in considerably overweight younger men. Duration and severity of obesity seem to limit the cardiovascular benefits of weight reduction in older men.

A Journal of Epidemiology and Community Health 2005;59:134-139. 